\title{
Estudio de los recursos educativos para público familiar en los museos de arte europeos y norteamericanos
}

\section{Study of the educational resources for familiar public in the European and North American museums of art}

\author{
Alma Obregón Fernández \\ Universidad Complutense de Madrid \\ aobregon@ccinf.ucm.es
}

Recibido: 21 de septiembre de 2010

Aprobado: 6 de abril de 2011

\begin{abstract}
Resumen
Hoy en día las familias no sólo constituyen un sector muy importante del público museístico, especialmente durante los fines de semana y vacaciones, sino que además son uno de los grupos más interesantes en lo que respecta a la educación en el museo, debido a las interacciones particulares que en él se producen y a las peculiaridades que, como grupo multigeneracional, le caracterizan. El objetivo de este artículo es analizar la trayectoria histórica de la programación educativa en los museos europeos y norteamericanos, prestando atención al momento en el que se introducen las familias como público objetivo de los mismos, para a continuación mirar en concreto al momento actual, atendiendo a los programas educativos que se destinan a familias en el marco de la programación didáctica general de un museo de arte y estableciendo una clasificación esquemática de los mismos.
\end{abstract}

Palabras Clave: Educación en el museo, Familias, Programación didáctica en el museo de arte.

Obregón Fernández, A. 2011: Estudio de los recursos educativos para público familiar en los museos de arte europeos. Arte, Individuo y Sociedad, 24(1), 9-20

\begin{abstract}
Nowadays, families not only make up a very important part of the total museum visitors, especially on weekends and holidays, but are also one of the most interesting groups when it comes to educational activities at the museum, due to the particular interactions that happen within each family and the peculiarities that are characteristic of a multi-generational group. The aim of this article is, firstly, to analyse the history of educational programs in European and North-American museums, focusing on the moment when families started to be considered a target audience, and then looking at the current time, in order to analyse the activities offered nowadays for families in art museums and educational programs and classify them in a diagram.
\end{abstract}

Key Words: Museum education, Families, Educational programs in museums of art.

Obregón Fernández, A. 2011: Study of educational resources for familiar public in ther European and North American museums of art. Arte, Individuo y Sociedad, 24(1), 9-20

Sumario: 1. Las raíces de la programación didáctica en el museo, 2. Las primeras experiencias en nuestro país, 3. Declive y renacimiento, 4. ¿Y las familias?, 5. Esquematización del panorama actual. Referencias 


\section{Las raíces de la programación didáctica en el museo}

Las labores educativas del museo, tal y como hoy las entendemos, y más allá de las intenciones de la Ilustración de difundir la cultura y la educación mediante la creación de los primeros museos públicos, nacen a mediados del s. XIX, en Inglaterra, de la mano de Sir Henry Cole y el Victoria \& Albert, museo en el que de forma pionera se establece una programación educativa enfocada al público general.

La creación del Victoria \& Albert Museum (bajo el nombre inicial de Museum of Manufactures) forma parte de las iniciativas del gobierno inglés por mejorar la calidad del diseño industrial ${ }^{1}$ y se enraíza en la clausura de la Exhibición Universal de 1851, cuyos beneficios son administrados con el fin de "aumentar los medios para la educación industrial y extender la influencia de la ciencia y el arte sobre las industrias productivas". ${ }^{2}$ Henry $\mathrm{Cole}^{3}$, su primer director, tiene un papel fundamental, pues pone en marcha el programa educativo, amplía las colecciones y promueve la catalogación exhaustiva de los objetos que las componían. Impulsado por la creencia anglosajona de la importancia de la responsabilidad cívica del museo de arte, el Victoria \& Albert se convierte en el primer museo que dirige programas educacionales de forma sistemática a una audiencia muy amplia y marca el origen de los programas educativos en los museos tal y como hoy los entendemos.

Las iniciativas de Cole van a propagarse con rapidez, no sólo mediante la creación de museos de artes decorativas para mejorar la calidad de diseño nacional ${ }^{4}$, sino sobre todo por la influencia de sus programas educacionales tanto en museos de artes decorativas como en museos con colecciones de distinta naturaleza ${ }^{5}$, llegando con especial fuerza a los Estados Unidos. Puede afirmarse que el museo norteamericano se plantea desde sus inicios un objetivo pedagógico activo para mejorar la cultura del pueblo $\mathrm{y}$, así, la mayor parte de los museos americanos inauguran programas educativos desde su creación (y algunos incluso antes ${ }^{7}$ )

\section{Las primeras experiencias en nuestro país}

En nuestro país hay destacan las iniciativas de los reformadores de la pedagogía a finales del s. XIX y principios del XX, como Manuel Bartolomé Cossío, el cual puso ya desde sus primeros escritos un énfasis en la importancia de enseñar a los niños "el arte de saber ver" ${ }^{8}$ y en la necesidad de generar en el niño un interés por aprender. ${ }^{9}$ Estas ideas se hicieron realidad mediante las iniciativas promovidas en el marco de la Institución Libre de Enseñanza. ${ }^{10}$ Cossío fue así el primero en España en realizar visitas a museos con niños. ${ }^{11}$

Otra iniciativa destacada es el Museo Pedagógico Nacional, cuya labor de formación del profesorado buscaba precisamente una actualización de los métodos educativos del momento, fomentando actividades fuera de la escuela (como, por ejemplo, las visitas a museos). Además, el Museo lanza las Misiones Pedagógicas, entre cuyas actividades se contaba con un Museo Circulante. ${ }^{12}$

Los efectos de todas estas iniciativas entre la población son palpables, como podemos ver reflejados en la descripción de esta nueva actitud ante el museo que realiza Eugenio D’Ors en Las Ideas y las Formas: “Empieza a ser conocido un cambio 
de actitud en las generaciones nuevas de curiosos, de estudiosos al acercarse a los museos. Acontece que hombres de hoy, especialmente los jóvenes y los niños, que tal vez en compañía de padres y profesores cumplen tales visitas se encaran con las obras de los grandes maestros, gustan de ellas enseguida y de sopetón las entienden [...] La sensibilidad de estos representantes del mundo nuevo parece encontrarse, previa y naturalmente, dispuesta a la penetración y a la simpatía ante las obras de los antiguos maestros. [...] Feliz situación espiritual la del niño novecentista que visita un museo". ${ }^{13}$

\section{Declive y renacimiento}

En todo caso, y pese a las iniciativas desarrolladas en España, en Europa la labor educativa del museo sufre un declive a principios del s. XX, en favor de un mayor interés de los conservadores de museos por sus colecciones. ${ }^{14}$ Finalmente es en los años 60 cuando se produce el boom de los programas educativos tanto en Europa como en Estados Unidos. Un estudio señala que en Estados Unidos, entre 1966 y 1974, el 51\% de los museos americanos expandieron su oferta educativa. ${ }^{15}$ En 1974, el 92\% de los directores de museos señalaba como su propósito más importante "proveer al público con servicios educativos". ${ }^{16}$

En España, la llegada de la democracia va acompañada de un nuevo interés de parte de los conservadores por conocer lo que se está haciendo en el extranjero. Las iniciativas más tempranas se ponen en marcha en el Museo Español de Arte Contemporáneo, y consisten en una serie de actividades para niños vinculadas a las colecciones hoy alojadas en el Museo Nacional Centro de Arte Reina Sofía. En estos años, la creación de centros de arte contemporáneo se acompaña del desarrollo de programas educativos que buscan acercar el mundo del arte contemporáneo a la población general. Paralelamente comienzan a darse iniciativas novedosas en museos de una historia larga y consolidada. Es el caso del Museo Arqueológico Nacional, cuyas educadoras, Angela García Blanco ${ }^{17}$ y Teresa Sanz Marquina, publican en 1979 un artículo llamado "El departamento educativo en el museo"18 en el que describen que una de sus funciones ha de ser "sacar el museo fuera de sus muros". ${ }^{20}$ Esta idea va a tener su puesta en práctica mediante iniciativas como las que se desarrollan en el Museo de Escultura de Valladolid (hoy Museo San Gregorio) que en 1983 lleva a cabo una experiencia pionera en nuestro país al crear la primera Maleta Pedagógica, titulada "Cómo se hace una escultura"19 o con las "Meninas Viajeras" del Museo del Prado en los años 90.

\section{4. ¿Y las familias?}

¿En qué momento pasan las familias a ser público destacado de las actividades educativas en el museo? Habiendo estado inicialmente enfocados en exclusiva a los sectores de la educación reglada (público escolar y universitario) y a público adulto (sobre todo al profesorado), en la última década los museos han tenido que ir abriéndose a nuevos públicos con otras características y necesidades, y en esta apertura las familias han pasado a ser las protagonistas en el museo de los programas educativos 
no vinculados a educación reglada. Así, aunque la educación en el museo de grupos escolares tiene una trayectoria de casi ciento cincuenta años, la atención a los niños que lo visitan en familia es bastante reciente. En el libro de 1989, Museums Visits and Activities for Family Life Enrichment, se detectaba que, entre 1950 y 1988, la revista Museum News había publicado tan sólo un artículo dedicado a la familia como público del museo, demostrando esta falta de atención específica a las familias. ${ }^{20}$

Es por tanto a partir de los años 90 cuando los educadores de los museos, conscientes de la importancia de las familias como público del museo, pero sobre todo como "unidad que persigue un aprendizaje", desarrollan cada vez más programas dedicados al público familiar. Esta programación alienta a las familias "a mirar las cosas desde diferentes perspectivas e interactuar de diferentes maneras". ${ }^{21}$ Hoy, las familias son un público destacado del museo y demandan una programación educativa acorde con sus características propias que les diferencian de otros públicos existentes en el museo. Y es que se trata de un grupo multigeneracional, pues el rango de edades presentes es muy amplio, y en el que el nivel sociocultural y económico puede ser muy variado, habiendo fuertes oscilaciones de conocimientos tanto entre padres procedentes de ambientes muy dispares, como en los hijos, de diferentes edades y contextos socioculturales.

Actualmente la mayoría de los grandes museos de arte ${ }^{22}$ cuentan con una sección de su programación y publicaciones enfocada directamente a las familias, si bien es detectable una mayor presencia y grado de desarrollo de estas propuestas en los museos anglosajones, estando Inglaterra y Estados Unidos a la cabeza en cuanto a número de propuestas, especialización y complejidad de las mismas, y público alcanzado.

\section{Esquematización del panorama actual}

Como puede verse en el esquema-resumen (fig.1) la oferta actual para familias puede clasificarse en dos áreas: herramientas para la visita autónoma y actividades guiadas en el museo. La presencia o no de estas dos áreas en la programación de un museo es uno de los parámetros que pone de manifiesto el estado de desarrollo de su actividad educativa para familias. ${ }^{23}$

La diferencia principal entre las dos áreas principales mencionadas es la intervención directa o indirecta de los educadores del museo. En el caso de las herramientas diseñadas para facilitar una visita autónoma, la participación de los educadores del museo es indirecta, pues lo hacen mediante los textos, actividades o audios preparados con antelación. ${ }^{24}$ En el caso de las actividades guiadas en el museo, éstas cuentan siempre con la participación de un educador del museo en algún momento de la actividad. Puede que su presencia no dure el periodo completo de visita al museo, pero esta figura es necesaria para llevar a cabo la actividad, que en ningún caso se puede realizar al completo de forma autónoma.

Comienzo por tanto por las actividades guiadas, entre las que podemos encontrar una gran variedad. En primer lugar, me centraré en la forma más básica: las visitas guiadas para familias, derivadas de las actividades educativas que se han venido desarrollando desde mucho tiempo atrás enfocadas a público escolar y adulto. Los itinerarios habituales se reducen y los contenidos se simplifican, buscando ser com- 
prendidos por los niños a la vez que despiertan el interés de los mayores mediante una dinámica intensa de preguntas y respuestas, fomentando la participación de los presentes.



Figura 1. Esquema elaboración propia

Por otro lado, esta modalidad ha evolucionado a variedades como las visitas teatralizadas o con danza. La pertinencia de la incorporación de estas variedades a la programación educativa de un museo depende de la capacidad didáctica de las mismas, pues en ocasiones se centran únicamente en la espectacularidad o en el entretenimiento y olvidan la transmisión de contenidos.

Otra forma que habitualmente toman estas actividades es la de cuentacuentos, que suele tener un contenido vinculado a las obras de la colección o de la exposición temporal para evitar limitarse a un mero entretenimiento que podría desarrollarse en cualquier otro entorno.

Las visitas-taller son otra categoría de actividades para familias que consiste en una visita a la colección que concluye con la realización de un taller en el que participan padres e hijos y mediante el que se profundiza en lo aprendido durante la visita. 
Muy comunes en el extranjero son los festivales y eventos centrados única y exclusivamente en las familias. Se trata de que, durante uno o varios días, las familias sean las protagonistas del museo, con numerosas actividades: talleres, visitas, cuentacuentos, concursos de arte, etc. ${ }^{25}$

Finalmente encontramos los Ciclos de cine para familias, en los que se programan una serie de documentales, cortos, películas, etc. de interés para niños y adultos.

Respecto a las herramientas para la visita autónoma, hay una característica común a todas ellas y es el uso de un doble nivel de lectura: unos textos se destinan a los padres, otros a los hijos. La tarea de los padres pasa a ser la de aplicar las propuestas de forma correcta y seguir unas pautas que les marcan los educadores mediante ese material. Esto les facilita mucho la labor de guiar a los niños por el museo y aporta garantías para que la visita sea exitosa.

Como en el caso de las actividades guiadas, en este ámbito son también muy variadas las propuestas. Los mapas familiares adaptan la información habitualmente esquemática de los mapas de museo a un lenguaje más cercano a lo que los niños comprenden. ${ }^{26}$

Las hojas informativas casi siempre se enfocan más a los padres, buscando aportar una serie de pautas para facilitar una visita adaptada a los niños, lo cual es difícil para muchos padres, acostumbrados a llevar a cabo las visitas siempre en compañía de adultos.

Las características de las hojas de actividades varían de unos museos a otros, pero generalmente se trata de que la familia realice una visita con un itinerario concreto (de 4 a 6 obras normalmente) y que pueda desarrollar una interacción con cada una de las obras que contempla, bien mediante el diálogo con preguntas y respuestas, o mediante la realización de una actividad vinculada a esa obra (escribir un texto sobre una temática relacionada con ella, dibujar alguno de sus elementos, etc.).

Semejantes a estas hojas, pero con más contenido y menos actividades, son los itinerarios para familias. Se trata de publicaciones breves, de 4 ó 5 páginas como mucho, en las que se desarrolla un itinerario adaptado al público familiar y en el que se plantean preguntas, curiosidades, pequeños juegos, etc. que facilitan las interacciones dentro del grupo familiar.

Una variedad próxima, aunque más extensa, son las guías para familias. El número de páginas es mayor, en torno a las 20 , y podrían describirse como conjunción de mapa familiar, hoja informativa, itinerario y folleto de actividades.

Otra variante son las mochilas con actividades..$^{27} \mathrm{La}$ primera diferencia con las herramientas previamente analizadas es que, en este caso, las mochilas (o materiales) se "alquilan" o se "toman prestadas" durante la visita y han de devolverse antes de abandonar el museo. La segunda diferencia, derivada precisamente de que los materiales se devuelvan al terminar la visita, es que este formato puede contar con materiales más elaborados y costosos (que serían imposibles de financiar si cada familia se los llevara a su casa).

Otra herramienta habitual en los museos anglosajones pero no tanto en el continente son las actividades en sala o "hands-on exhibits" (exposiciones manuales) que consisten en una actividad manual paralela a las obras que se exponen en esa sala. ${ }^{28}$ 
Entre las herramientas multimedia, hay un recurso que cada vez se extiende más: el de las audioguías infantiles, que en algunos casos se configuran como guías multimedia. Estas audioguías presentan contenidos adaptados a los más pequeños y les conducen por un itinerario pensado para niños. ${ }^{29}$

Dentro de los elementos multimedia se encuentran también las aplicaciones interactivas en sala. Estos recursos, parte esencial de la mayor parte de museos de ciencia y tecnología, son poco habituales en los museos de arte aunque poco a poco se van instalando. ${ }^{30}$

Muchos museos ofrecen además juegos y actividades online vinculados a sus colecciones que pueden realizarse desde casa antes o después de la visita. ${ }^{31}$

Finalmente, otro recurso muy interesante son los cuentos ${ }^{32}$ inspirados en obras de la colección que, especialmente en el caso de niños muy pequeños, sirven para continuar en casa con la introducción de los niños en el mundo del arte que comienza en el museo.

\section{Referencias}

Alexander, E. P. (1995). Museum masters: their museums and their influence. Maryland: Rowman Altamira.

Arnaldo, J. (2009). Actas I Congreso Internacional. Los museos en la educación. Madrid: Museo Thyssen-Bornemisza.

Baker, M., Burton, A y Richardson, B. (Ed.). (1997). A Grand Design: The Art of the Victoria and Albert Museum. Londres: V \& A Publications.

Beaumont, E y Sterry, P. (2005). A study of grandparents and children as visitors to museums and art galleries in the UK. Museum and society, 3(3), pp. 167-180.

Benlloch, M. y Williams, V. N. (1998). Influencia educativa de los padres en una visita al museo de la ciencia: Actividad compartida entre padres e hijos frente a un módulo. Enseñanza de las ciencias, 16(3), pp. 451 - 460.

Black, G. (2005). The Engaging Museum. Developing museums for visitor involvement. Londres: Routledge.

Buttler, B. H. y Sussman, M. B. (Ed.). (1989). Museum Visits and Activities for Family Life Enrichment. Londres: Routledge.

Charman, H. (2005). Uncovering Professionalism in the Art Museum: An Exploration of Key Characteristics of the Working Lives of Education Curators at Tate Modern. Tate Papers, primavera. Recuperado de http://www.tate.org.uk/research/tateresearch/tatepapers/05spring/charman.htm

Cox, A., Lamb, S. y Orbach, K. (2000) A shared experience: A qualitative evaluation of family activities at three Tate Sites. Informe interno de la Tate.

De Frutos González, E. (2006). Actividades para niños y familias en el Museo Nacional del Prado. El área de educación (2003-2005). MUS-A, IV(6), pp. $70-73$.

Dobbs, S. M. (1971). Dana and Kent and early museum education. Museum News, 50(2), pp. $38-41$. 
Falk, J., Moussouri, T. y Coulson, D. The Effect of Visitors. Agendas on Museum Learning. Curator, 41(2), pp. 106 - 120.

García Blanco, A. (1988). Didáctica del museo. El descubrimiento de los objetos. Madrid: Eds. de la Torre.

García Blanco, A. y Sanz Marquina, T. (1979). El departamento educativo en el museo. Boletín de la ANABAD, 29(4), pp. 45 - 49.

García Rubio, A. I. (2000-2001). La Maleta Pedagógica: un proyecto educativo para el Museo Nacional de Machado de Castro. Imafronte, 15, pp. 85 - 102.

Moffat, H. y Woollard, V. (Ed.). (1999). Museum \& Gallery Education. Londres: The stationery office.

Harris, Q. (1997). Children as an Audience for museums and galleries. Informe preparado para el UK Arts Council and Museums and Galleries Commission.

Hein, G. H. (1998). Learning in the museum. Londres: Routledge.

Hooper-Greenhill, E. (1998). Los Museos y sus Visitantes. Gijón: Trea.

Kelly, L., Savage, G., Griffin, J. y Tonkin, S. (2004). Knowledge Quest: Australian Families Visit Museums. Sidney y Canberra: Australian Museum and National Museum of Australia.

León, A. (1990). El museo. Teoría, praxis y utopía. Madrid: Cátedra.

Miles, R. y Zavala, L. (Ed.). (1994). Towards the museum of the future. London: Routledge.

Newsom, B.Y. y Silver, A. Z. (Ed.). (1978). The Art Museum as Educator: a collection of studies as guides to practice and policy. California: University of California Press.

Rawlins, K. (1978). Educational Metamorphosis of the American Museum. Studies in Art Education, 20(1), pp. 4 - 17.

Ruddock, P. (2009). V\&A Annual Review 2008/2009. Londres: Victoria \& Albert Museum.

Solinger, J. W. (Ed.). (1990). Museums and Universities: common continuing education museums and constituencies. Nueva York: Macmillan.

Sterry, P. y Beaumont, E. Methods for studying family visitors in art museums: A cross-disciplinary review of current research. Museum Management and Curatorship, 3(21), pp. 222 - 239.

Valdes Sagües, M. del C. (1999). La difusión cultural en el museo: servicios destinados al gran público. Gijón: Trea.

Wittmann, O. (1966). The Museum and Its Role in Art Education. Art Education, 19(2), pp. $3-6$.

\section{Notas}

1. "The V\&A [...] had, from its foundation, a unique mission - the education of designers in the principles of design; the education of the public as consumers of design; and the education of young people as the designers and consumers of the future". RUDDOCK, Paul.: V\&A Annual Review 2008/2009. Londres: Victoria \& Albert Museum, 2009, p. 6. 
2. Con este objetivo, la Comisión Real encargada de la administración de beneficios compra $350.000 \mathrm{~m} 2$ en South Kensington en los cuales se situarán más tarde los tres grandes museos (Victoria \& Albert, Historia Natural y Ciencia), el Royal Albert Hall y otras instituciones educativas como el Imperial College y los Royal Colleges de Arte y Música.

3. Henry Cole (1808-1882) había dirigido el Department of Practical Art desde 1852 y se mantuvo en el puesto hasta 1873, pese al cambio de nombre a Department of Science and Art. Además, había sido administrador jefe de la Comisión Real organizadora de la Exhibición de 1851.

4. Henry Cole afirmaba ya en 1874 que el modelo del South Kensington Museum había servido como inspiración a 35 museos en todo el mundo, aludiendo a los museos de artes decorativas creados en Viena, Karlsruhe, Berlín, Hamburgo, etc. [ALEXANDER, E. P.: Museum masters: their museums and their influence. Maryland: Rowman Altamira, 1995, p. 166]

5. ALEXANDER, E. P.: op. cit., p. 166.

6. LEÓN, A.: El museo. Teoría, praxis y utopía. Madrid: Cátedra, 1990, p. 53.

7. Como curiosidad, el Museo de Cleveland empezó su programa educativo un año antes de su inauguración en 1915. Lo mismo sucedió con el Museo de Toledo en 1903. En SILVER, A. Z.: "Issues in Art Museum Education: A Brief History", en NEWSOM, B. Y.; SILVER, A. Z. (eds.): The Art Museum as Educator: a collection of studies as guides to practice and policy. California: University of California Press, 1978, p. 13.

8. ARNALDO, J.: "Pedagogía de la vanguardia. El descubrimiento artístico de la revolución educativa" en ARNALDO, J. (dir.): Actas I Congreso Internacional. Los museos en la educación. Madrid: Museo Thyssen-Bornemisza, 2009, p. 32.

9. En su Notas sobre construcción escolar de 1900, señala que el aula debe representar para el niño "un sitio donde afirmar, ordenar y construir las ideas y datos recogidos en el campo, en el museo, en la fábrica, en la sociedad, en medio de la vida, en suma, a donde hay que llevar la escuela, porque es donde se aprende: ante los objetos y ante los hechos capaces de despertar el interés y engendrar la educación activa”. En COSSIO, M. B.: Notas sobre construcción escolar. Madrid: R. Rojas, 1900, p. 2-3.

10. El crítico de arte Moreno Villa, que asistió a una de sus visitas al Prado con alumnos de la ILE, la describía así: "Eran lecciones vivas que iban naciendo y creándose ante las dudas o preguntas de los alumnos y las reacciones del profesor, o bien ante las preguntas de éste y la reacción del alumno. Algo muy parecido a los coloquios socráticos”. Palabras del crítico de arte Moreno Villa recogidas en OTERO URTAZA, E.: Manuel Bartolomé Cossío: pensamiento pedagógico y acción educativa. Madrid: Centro de Publicaciones del Ministerio de Educación y Ciencia, 1994, p. 73.

11. ARNALDO, J.: op. cit., p. 35-36.

12. Éste estaba formado por dos colecciones de catorce cuadros cada una que se exponían durante una semana en localidades de tamaño medio e iban acompañadas de las charlas explicativas de los "misioneros" y del regalo de copias fotográficas de las pinturas para ser colgadas en escuelas y centros recreativos populares. BOLAÑOS, M.: Historia de los museos en España. Gijón: Trea, 1997, p. 353.

13. Palabras recogidas de Las ideas y las formas de Eugenio D’Ors. Citadas por BOLAÑOS, M.: op. cit., p. 336. 
14. Los museos europeos, contando ya con grandes colecciones en edificios muy señalados, quedan en esos momentos satisfechos con la protección de las mismas y su puesta a disposición del público. Por su parte, los museos americanos, ante la carencia inicial de colecciones importantes, apuestan por la labor educativa y de difusión. VALDÉS SAGÜÉS, M. del C.: La difusión cultural en el museo: servicios destinados al gran público. Gijón: Trea, 1999, p. 34; HEIN, G. H.: Learning in the museum. Londres: Routledge, 1998, p. 5; y WITTMANN, O.: "The Museum and Its Role in Art Education". Art Education, vol. 19-2, 1966, p. 4.

15. RAWLINS, K.: "Educational Metamorphosis of the American Museum", en Studies in Art Education, vol. 20 - 1, 1978, p. 9.

16. National Endowment for the Arts. Museums U. S. A. Washington, D. C.: U. S. Government, 1974.

17. Su libro Didáctica del museo sería un manual destacado para los educadores de museos en España. Ga BLANCO, A.: Didáctica del museo. El descubrimiento de los objetos. Madrid: Eds. de la Torre, 1988.

18. GARCÍA BLANCO, A.; SANZ MARQUINA, T.: "El departamento educativo en el museo", Boletín de la ANABAD, Tomo 29, no 4, 1979, pp. 45-49.

19. DE LOS ÁNGELES, M.: "Una maleta de préstamo", Cuadernos de pedagogía, no 134, 1986, pp. 25-27.

20. BUTTLER, B. H. y SUSSMAN, M. B. (eds.): Museum Visits and Activities for Family Life Enrichment. Londres: Routledge, 1989, p. 3.

21. HOPPENFELD, S.: "Families and Art Galleries: How Values and Philosophies Shape Spaces". Ponencia presentada en el simposio del J. Paul Getty Museum: "From Content to Play: Family - Oriented Interactive Spaces in Art and History Museums" celebrado los días 4 y 5 de junio de 2005 (Actas disponibles online en http://www.getty.edu/education/ museum_educators/index.html Último acceso 8/3/2010)

22. Hay que señalar que, aunque es en los museos de ciencia donde más se ha trabajado en los programas educativos para familias, la mayor parte de las veces es difícil aplicar sus logros al campo de los museos de arte, pues las diferencias entre las colecciones que estos museos alojan y las que podemos encontrar en un museo de arte no podrían ser mayores. Una diferencia clave es el papel de la obra expuesta respecto al discurso del museo. En el museo de arte la protagonista es la obra de arte. Son las propias obras las que suscitan la organización que propone el conservador, y son también las que dan pie a la descripción que las acompaña en la cartela, sin que la ausencia de datos sobre el autor y lugar/fecha de ejecución pudiera ser impedimento para disfrutar la obra y aprender de ella. En el museo de ciencias, sin embargo, encontramos generalmente que el objeto ejerce de ejemplo del tema que se está exponiendo en la sala. En otras ocasiones, además, los ejemplos necesarios tienen características que los hacen imposibles de musealizar (como un volcán o un terremoto), lo que obliga al conservador a valerse de maquetas, aplicaciones interactivas, simuladores, etc. De ahí que en este tipo de museos se haya ahondado mucho más en la faceta didáctica, ya que es fundamental hacer comprender lo que se expone y para ello se usan todos los recursos disponibles: numerosas cartelas y paneles explicativos, dispositivos interactivos, vídeos, simuladores, guías didácticas, juegos, charlas, itinerarios, etc. 
23. En general, en los museos con una trayectoria más corta en este ámbito o, mejor dicho, con una menor dedicación a este público, encontraremos o bien una ausencia de las herramientas para la visita autónoma o que éstas son muy limitadas, mientras que lo habitual es que las actividades guiadas estén presentes en la gran mayoría de los museos de arte con un mayor o menor grado de intensidad y/o complejidad. En los museos con una gran dedicación de tiempo y presupuesto al ámbito familiar, las actividades guiadas en el museo se complementan con una amplia variedad de herramientas para la visita autónoma. En este sentido, hay que tener en cuenta que el hecho de que un museo sea de creación reciente no es un factor que determine el grado de desarrollo de esta área educativa, pues muchos de los museos más recientes (como Artium o el Musac en nuestro país, o el Musée du Quai Branly en París) cuentan con una fuerte programación educativa, estando una importante parte de ella dedicada a las familias.

24. Estas herramientas pueden diseñarse tanto para su utilización dentro del museo durante la visita como para su uso previo (con vistas a preparar la visita) o a posteriori (para recordar lo vivido y reforzar lo aprendido)

25. Un museo que realiza esto de forma habitual es el Victoria \& Albert Museum, que cuenta con fines de semana especialmente dedicados a las familias en los que hay talleres, ciclos de cine, visitas guiadas, etc. En nuestro país estos eventos no son tan habituales, pero sí que se han realizado con motivos como el Día de la Familia.

26. Se incluyen dibujos y gráficos que proporcionan más información que un mero "Sala 12 " y normalmente incluyen la normativa del museo de una forma que sea "divertida" para los niños (¡Recuerda a tus padres que no pueden tocar las obras!). Destaca por ejemplo el del Metropolitan Museum de Nueva York, a todo color y con ilustraciones muy divertidas.

27. Hago referencia a "mochilas" por ser el formato más habitual. Sin embargo puede adoptar otras formas, como los "cinturones de herramientas" (como en la Galería de Arte de Manchester) en los que se proporcionan una lupa, binoculares, pistas, etc. para que los niños exploren las galerías como si fueran detectives. En otros casos, no se ofrece una mochila en sí, pero sí un conjunto de materiales diseñados para realizar una actividad concreta, como cuadernos, pinturas, etc.

28. Por poner un ejemplo, en el V\&A Museum es posible que los niños se prueben unas enaguas y una capa en las British Galleries, para así comprender mejor las salas del s. XIX, así como construir un "palacio de cristal" con bloques de metacrilato, en referencia a la Gran Exhibición de 1851. En las galerías relativas al teatro, recientemente inauguradas, se ofrece la posibilidad de probarse diferentes disfraces de personajes teatrales a niños y mayores.

29. El problema básico de las audioguías es que puede restringir la interacción adulto - niño (por limitarse cada uno de ellos a escuchar su audioguía sin más). Una idea para evitar este problema es la que han puesto en práctica en la National Gallery de Londres, ya que en una de sus guías familiares los padres han de quitarse los auriculares mientras los niños oyen "secretos" que luego tienen que contar a sus padres. 
30. Por ejemplo, en algunas de las salas de la sección Wonderkamers del Gemeentemuseum de La Haya no existen cartelas, sino que hay que consultar las pantallas interactivas para conocer los detalles del objeto. Los beneficios son muchos si los contenidos son los adecuados, pues permite ampliar la información sobre los objetos presentes sin ocupar el espacio de un letrero y además permite alojar datos adaptados a las diferentes edades.

31. En ocasiones son aplicaciones interactivas, otras veces se trata de material descargable que hay que imprimir (recortables, manualidades, hojas coloreables...) Este tipo de herramientas son muy útiles, pues requieren una supervisión paterna, tanto si se trata de un programa interactivo como si es una manualidad, y permite continuar con la labor educativa del museo en la propia casa.

32. Por ejemplo, el Metropolitan Museum de Nueva York cuenta entre sus publicaciones con un cuento para niños cuyo argumento hace referencia al cuadro de su colección "Dish of apples", pintado por Paul Cézanne (ca.1875-77) 